\title{
The. \\ Laryngology \\ ENT trainee papers presented at the ENT \& Otology \\ Scotland Winter Meeting, 8 November 2019, Stirling, Scotland, UK
}

cambridge.org/jlo

\section{Online Only Abstract Selection}

Cite this article: . ENT trainee papers presented at the ENT Scotland Winter Meeting, 8 November 2019, Stirling, Scotland, UK. J Laryngol Otol 2021;E1:1-5. https://doi.org/ $10.1017 /$ S0022215121001122

First published online: 6 May 2021

\author{
Non-technical skills for surgeons and ear nose and throat surgery \\ R Bannon, K Stewart and M Bannister \\ From Victoria Hospital, Kirkcaldy
}

Aims

Surgeons' non-technical skills complement their surgical abilities and are related to patient outcomes. The non-technical skills for surgeons behaviour rating system is wellestablished in general surgery and human factors training. We present a study assessing and evaluating the application of non-technical skills for surgeons to otolaryngology surgery.

\section{Methods}

A prospective study of three otology operations over three months analysing the four elements of non-technical skills for surgeons taxonomy is presented. Good and poor behaviours were recorded, learning points identified and improvement strategies employed.

\section{Results}

Non-technical skills for surgeons' ratings improved for all three operations during the study period. Consultant and trainee ratings were similar throughout the study period. The 'decision making' and 'leadership' elements showed the greatest improvement. 'Situation awareness' improved the quickest, whereas 'communication and teamwork' improvement was slower.

\section{Conclusions}

Applying non-technical skills for surgeons improved surgical flow and operation theatre usage, particularly as operation theatre personnel regularly rotated. Non-technical skills for surgeons application may have similar benefits in more demanding skull base and oncological resection and reconstruction surgery.

Computed tomography scan features as predictors of sinonasal inverted papillomas: a local case series

F Cooper, A Wain Yong and S Maini

From Aberdeen Royal Infirmary, Aberdeen

\section{Aims}

Early recognition of inverted papillomas can aid surgical planning to help reduce the risks of recurrence and malignant transformation. This study aimed to determine the rates of presence of certain computed tomography (CT) scan features of sinonasal inverted papillomas and their correlation with outcomes.

\section{Methods}

This study was a retrospective analysis, with input from radiology, of pre-operative CT scan features and outcomes of a case series of patients with inverted papillomas managed surgically over a five-year period in one centre.

\section{Results}

Twenty-four cases were identified. Fifty per cent of cases had bony resorption or destruction, and 29 per cent of cases had an area of focal hyperostosis identifiable on the pre- 
operative CT scan. Intra-lesional calcifications were rarely seen. Revision surgery was required in 42 per cent of cases, with 60 per cent of these cases displaying radiological features on the original CT scan.

\section{Conclusions}

Presence of certain CT scan features may represent more aggressive tumours. Recognising these features is important for surgical planning. This case series is limited by size and heterogeneity.

Total thyroidectomy for Grave's disease: what do our patients think? A qualitative cohort study to evaluate the surgical management of Grave's disease

L Hamilton, A E Lim and L Clark

From Queen Elizabeth University Hospital, Glasgow

Aims

Total thyroidectomy is an established treatment for Grave's disease. The literature covers physical complications but has not focused on patient opinion. The authors of this study ask what our patients really think and whether there is significant improvement in quality of life post-operatively.

\section{Methods}

Ninety-five patients who underwent total thyroidectomy for Grave's disease between 2015 and 2018 were identified. Fifty-four responded to a phone-call questionnaire. Questions were based on the Glasgow Benefit Inventory for patients before and after surgery.

\section{Results}

Average Glasgow Benefit Inventory scores were 13.35 (standard deviation $(\mathrm{SD})=30.16)$. A general subset had an average score of $19.21(\mathrm{SD}=32.59)$, a social subset average was 9.57 $(\mathrm{SD}=25.61)$ and a physical subset was average $-6.79(\mathrm{SD}=$ 37.40). Patients were asked for their feedback on pre-operative communication and post-operative side effects.

\section{Conclusions}

Pre-operative communication was of paramount importance. Overall, the Glasgow Benefit Inventory scores were positive, showing an improvement in quality of life since having total thyroidectomy for Grave's disease.

\section{Intracranial complications of otorhinolaryngological infections: changing trends and management}

F Ng Kee Kwong ${ }^{1}$, A Makepeace ${ }^{1}$, G Irwin ${ }^{1}$, C Doherty ${ }^{1}$, R Sangra $^{1}$, S Okhovat ${ }^{2}$ and W A Clement ${ }^{1}$

From ${ }^{1}$ Royal Hospital for Children, Glasgow and ${ }^{2}$ Queen Elizabeth University Hospital, Glasgow

\section{Aims}

This study aimed to assess changes in time relating to the management and prevalence of intracranial complications arising from primary otorhinolaryngological infections in a tertiary paediatric hospital setting.

\section{Methods}

This study was a retrospective case note review of all children admitted to the Royal Hospital for Children, Glasgow, who underwent surgery for primary rhinological or otological infections with intracranial complications between 2012 and 2018.

\section{Results}

Fifty-six children underwent surgery for rhinological or otological associated infections. Twenty-eight cases of intracranial complications were identified. Twenty-one cases ( 75 per cent) were of ontological origin and 7 cases ( 25 per cent) were of rhinological origin. The male-to-female ratio was $2.5: 1$, and the mean age was 7.2 years (range, 0.2-15.2 years). Twenty-two patients (78.6 per cent) required otorhinolaryngological surgical intervention alone with 6 patients $(21.4$ per cent) requiring combined otorhinolaryngological and neurosurgical surgery. During the study period the incidence of intracranial complications increased from 29.2 per cent to 65.6 per cent $(p=0.01)$.

\section{Conclusions}

The frequency of intracranial complications of primary otorhinolaryngological infections appears to be increasing over time.

An open structure questionnaire on reasons for delay in presentation: a prospective study of patients attending clinic with red flag symptoms of head and neck cancer

\author{
A E Lim and J Montgomery \\ From Queen Elizabeth University Hospital, Glasgow
}

Aims

The reasons for delayed presentation in cancer cases are reported as patient or professional delays. Studies have examined patient demographic factors associated with presentation delay, but it is currently unknown why patients feel they delayed presentation.

\section{Methods}

This was a prospective questionnaire study of consecutive patients attending an urgent suspicion of cancer clinic at a tertiary referral centre in Glasgow over a three-month period.

\section{Results}

A total of 395 patients were included with 164 patients (41 per cent) who waited up to 4 weeks, 139 patients ( 35 per cent) who waited up to 6 months and 31 patients ( 8 per cent) who waited longer than 6 months before presenting. There were 123 patients (31 per cent) who were not worried about their 
symptoms. Five of the 13 patients (38 per cent) diagnosed with head and neck cancer waited up to 6 months before presenting. Five patients with cancer (38 per cent) delayed presentation because they were not worried about their symptoms.

\section{Conclusions}

Reason for patients with head and neck cancer to delay their presentation is because they are not worried about their symptoms, possibly contributing towards later stage diagnosis.

Post-tonsillectomy haemorrhage after the introduction of intravenous antibiotics at induction in NHS Lanarkshire

A Loroch, D Chakravarty and M Yaneza

From University Hospital Monklands, Airdrie

\section{Aims}

The NHS Lanarkshire morbidity and mortality meetings in 2017 noted an increasing rate of post-tonsillectomy haemorrhage regardless of operating surgeon or technique used. The rate for April to August 2017 was 32 per cent in comparison with 11.5 per cent in 2014 to 2015 . It was therefore deemed necessary to implement a test of change in order to prevent these complications. Intra-operative antibiotic use in patients more than 16 years old was assessed as a means to reduce the rate of post-tonsillectomy haemorrhage.

\section{Methods}

This was a retrospective data analysis using clinical coding to compare 10-month periods pre- and post-introduction of an intra-operative antibiotic.

\section{Results}

The post-tonsillectomy haemorrhage rate since the introduction of intra-operative antibiotics was 14.3 per cent.

\section{Conclusions}

Since the introduction of an intra-operative dose of intravenous antibiotics for tonsillectomy patients more than 16 years old, the post-operative haemorrhage rate decreased in NHS Lanarkshire.

Use of overnight pulse oximetry and the Snoring, Troubled-breathing, Unrefreshed Questionnaire for paediatric obstructive sleep apnoea in a district general setting

A Rogers, S Benzie, T Geary and L Fraser

From University Hospital Crosshouse, Kilmarnock

\section{Aims}

This study aimed to assess our indications for overnight pulse oximetry and the impact on subsequent ENT management. We assessed correlation of Snoring, Troubled-breathing,
Unrefreshed Questionnaire score with overnight pulse oximetry values in these patients.

\section{Methods}

This was a retrospective review of 32 overnight pulse oximetry reports over a 7-month period. Data collected included age, weight, indication, mean oxygen saturation, number of dips more than 4 per cent, outcome and Snoring, Troubled-breathing, Unrefreshed Questionnaire score.

\section{Results}

The commonest indication $(n=19)$ for overnight pulse oximetry was to assess severity of obstructive sleep apnoea (OSA) in children less than 3 years or those with significant comorbidity in order to determine the best setting for surgery. Fifty per cent were ultimately managed in a tertiary centre. Ten underwent overnight pulse oximetry because of diagnostic uncertainty but significant parental concern; these cases were all normal and parents were reassured. Snoring, Troubledbreathing, Unrefreshed Questionnaire score correlated poorly with the number of dips of more than 4 per cent $(r=0.0109)$ and mean nadir more than 4 per cent $(r=0.0031)$. Weight was poorly recorded in 19 per cent.

\section{Conclusions}

Referrals were generally appropriate and influenced management in all cases. Snoring, Troubled-breathing, Unrefreshed Questionnaire scores were insufficiently sensitive to stratify severity of OSA. A new protocol should be developed.

Suspected sleep disordered breathing in children: how is it actually being managed in the out-patient department of a large UK children's hospital?

A Sheldon and H Kubba

From Royal Hospital for Children, Glasgow

\section{Background}

Sleep disordered breathing is a common condition in children. We aimed to identify the proportion of our out-patient clinic consultations that focused on this condition and how these patients were being managed.

\section{Methods}

All patients seen in out-patient clinics in September 2018 and January 2019 were identified. Demographic data, clinic type, diagnostic focus of consultation and outcome of consultation were recorded.

\section{Results}

A total of 1505 patients were identified over 155 half-day clinics. Sleep disordered breathing was second only to hearing as most frequent focus of consultation (239 patients; 15.8 per cent). There were 159 patients who were new referrals and 80 who were review cases. Ninety-two (38 per cent of 239) were 
listed for surgery of whom 61 (66 per cent of 92) had no prior sleep investigations.

\section{Conclusions}

Sleep disordered breathing represents a significant proportion of the department's workload, for which a wide range of management strategies are used. Consistency could be improved with an evidence-based guideline regarding the use of sleep investigation.

\section{Operation theatre observer telescope: preliminary experience}

Kir Stewart, R Bannon and M Bannister

From Victoria Hospital, Fife

\section{Aims}

Open techniques in head and neck surgery can have limited operative field visualisation for observers. We developed an operation theatre observer telescope allowing non-operators to view and appreciate open surgical anatomy and techniques. We assessed the experience of observers and operators and discussed the surgical education benefits of the operation theatre observer telescope.

\section{Methods}

This was a prospective assessment of operation theatre observer telescope use by observers and operators using an objective visual analogue scale questionnaire and qualitative feedback.

\section{Results}

Assessments were performed in 21 operations. All observer grades reported improved views of surgery with operation theatre observer telescope. Non-consultant observers gained the greatest benefit in understanding anatomy and surgical practices. No operators reported that use of the operation theatre observer telescope disturbed concentration or operative flow.

\section{Conclusions}

The mirror component is more useful in observing facial plastic surgery. The telescopic component is better suited to open procedures. The operation theatre observer telescope allows consultants to safely observe trainees at a distance, providing greater autonomy and improving confidence. Adaptions have been made to improve user handling.

Substance abuse is associated with earlier presentation and poorer functional outcome in laryngeal cancer

N Woodley, T Ton, M A Mohd Slim, J Montgomery and C Douglas

From Queen Elizabeth University Hospital, Glasgow
Aims

Opioid use has been implicated as an independent risk for laryngeal cancer. We aimed to evaluate the disease burden in laryngeal cancer due to illegal substance abuse.

\section{Methods}

This was a retrospective analysis of patients diagnosed with laryngeal cancer from January 2013 to December 2017.

\section{Results}

A total of 329 patients were included with 39 who reported substance abuse (11.9 per cent), 20 (6 per cent) who used opioids, 12 (3.6 per cent) who used cannabis, 5 (1.5 per cent) who used cocaine and 20 ( 6 per cent) who used benzodiazepines. Laryngeal cancer patients with substance abuse were younger than those without substance abuse (mean: 53 (standard deviation (SD): 11.2) vs 66.5 (SD 9.9), respectively; $p<0.0001$ ) with higher tumour stage (mean $3.3 \mathrm{vs}$ 2.54 , respectively; $p<0.0001$ ) and higher incidence of supraglottic tumours (61.5 vs 43.1 per cent, respectively; $p=$ $0.0305)$. There was no significant difference in recurrence, mortality, time to recurrence or survival. Patients with substance abuse had a higher rate of tube feeding than those without substance abuse (69.2 vs 47.9 per cent, respectively; $p=0.0126)$ and laryngectomy (46.1 vs 23.4 per cent, respectively; $p=0.0025)$.

\section{Conclusions}

Substance abuse is associated with laryngeal cancer in patients who are younger, with a more advanced stage at diagnosis and a higher rate of laryngectomy.

\section{Pan-Scotland post-tonsillectomy haemorrhage rates}

T Milner, O Hilmi, J Marshall and K MacKenzie

From Queen Elizabeth University Hospital, Glasgow

\section{Aims}

Post-tonsillectomy haemorrhage is the most significant complication following tonsillectomy. The UK National Prospective Tonsillectomy Audit conducted in 2003 to 2004 reported a 3.5 per cent haemorrhage rate and a 0.9 per cent rate of return to the operation theatre. This study was conducted to assess post-tonsillectomy haemorrhage rates across Scotland.

\section{Methods}

Tonsillectomy outcome data were collated for all patients undergoing tonsillectomy in Scotland between 2013 to 2017. Using Information Services Division Scotland retrospective data, the 30-day readmission and 30-day return to operation theatre rates allowed an assessment of post-tonsillectomy haemorrhage rates. Data were validated through comparison with an audit conducted in NHS Greater Glasgow and Clyde between 2015 and 2016 . 


\section{Results}

Tonsillectomy was performed in 23429 patients during the period, with a mean of 2594 paediatric and 2092 adult tonsillectomies per year. The 30-day readmission rates were 12.1 per cent in paediatric patients and 19.9 per cent in adult patients, and the 30-day return to operation theatre rates were 1.7 per cent and 4.9 per cent, respectively. Readmission and return to operation theatre rates were similar across Health Boards for both adult and paediatric tonsillectomies.

\section{Conclusions}

Not only are the 30-day readmission and return to operation theatre rates significantly higher than in the National Prospective Tonsillectomy Audit, but they are also higher than any other reported series to date. Although male sex and operative techniques appeared to significantly affect posttonsillectomy haemorrhage rates in our local series, it would seem unlikely that this would explain the apparent five-fold increase in the rates. Furthermore, these tonsillectomy outcomes were similar across all health boards in Scotland, highlighting the need for detailed audit of current practice. Of most significance are the implications for patient consent for tonsillectomy.

Patient outcomes following paediatric naso-lacrimal surgery leading to the proposal of a new patient pathway for children presenting with epiphora

\section{P Sooby and D Wynne}

From Royal Hospital for Children, Glasgow

\section{Aims}

Naso-lacrimal duct obstruction in children is the most common cause of epiphora in children, with 1-6 per cent of the population becoming symptomatic. Management options include probe and syringing, stent insertion, and endonasal and external dacryocystorhinostomy. This study aimed to produce an evidence-based patient pathway for children presenting with epiphora.

\section{Methods}

This was a retrospective analysis of outcomes of all patients under the age of 16 years who had undergone surgery for naso-lacrimal duct obstruction between January 2007 and November 2017.

\section{Results}

A total of 455 operations were examined. Primary probe and syringing had a success rate of 83.5 per cent. Primary endonasal dacryocystorhinostomy had a success rate of 71 per cent and revision surgery was 82 per cent. External dacryocystorhinostomy had a success rate of 91 per cent. Patients in the dacryocystorhinostomy group had an average of 1.93 procedures prior to dacryocystorhinostomy.

\section{Conclusions}

We put forward a patient pathway where primary intervention is by probe and syringe followed by endosnasal dacryocystorhinostomy (and revision endonasal dacryocystorhinostomy) and then by external dacryocystorhinostomy. 